\title{
Outpatient prescribing pattern for acute bronchitis in primary healthcare settings in China
}

\author{
Mengyuan Fu ${ }^{1,2}$, Haishaerjiang Wushouer ${ }^{1,3}$, Lin $\mathrm{Hu}^{1}$, Nan Li $\mathbb{C}^{4}$, Xiaodong Guan $\mathbb{D}^{1,3,5 凶}$, Luwen Shi ${ }^{1,3,5 凶}$ and Dennis Ross-Degnan ${ }^{2}$
}

Inappropriate prescribing for acute bronchitis in primary healthcare settings (PHSs) is commonly seen worldwide. Here we describe the prescribing patterns and antibiotic use for acute bronchitis in PHSs across China. We conduct a nationwide crosssectional survey to collect outpatient prescriptions from PHSs in 2017. Patients diagnosed with acute bronchitis without other infections are eligible for this study. Generalized estimating equations are used for analysis. Overall, 10,678 prescriptions for acute bronchitis from 214 institutions are included. The antibiotic prescription rate is $44.5 \%$ for total prescriptions, and differs significantly by region and urban/rural status $(p<0.05)$. Among all single-antibiotic prescriptions, $91.5 \%$ are broad-spectrum. Two-thirds of the prescriptions contain medicines for symptom management. The overall guideline compliance rate of acute bronchitis treatment for adults is $31.0 \%$. Prescribing antibiotics, especially broad-spectrum ones, for acute bronchitis is commonly observed in Chinese PHSs. Targeted interventions are urgently needed for Chinese primary clinicians, especially in western rural areas.

npj Primary Care Respiratory Medicine (2021)31:24; https://doi.org/10.1038/s41533-021-00234-y

\section{INTRODUCTION}

Acute bronchitis, an inflammation of the lower respiratory tract manifested by acute cough, is among the commonest clinical condition responsible for primary healthcare consultations ${ }^{1,2}$. It is estimated that every year, $5 \%$ of the general population report an episode of acute bronchitis, $90 \%$ of whom seek medical advice ${ }^{3}$. Acute bronchitis is a self-limited condition caused by virus in at least $90 \%$ of cases, in which antibiotics have no role in treatment ${ }^{4,5}$. However, studies show that most patients with acute bronchitis are treated with inappropriate or ineffective therapies ${ }^{6}$. Antibiotics are inappropriately prescribed for acute bronchitis up to $70 \%$ of the time, accounting for $44 \%$ of all outpatient antibiotic prescriptions in the US ${ }^{4}$. Unnecessary antibiotic prescriptions provide no clinical improvement ${ }^{3}$, increase the prevalence of antibiotic resistance ${ }^{7}$, and healthcare costs ${ }^{8}$. Symptoms of acute bronchitis, such as acute cough and sputum production, generally last for $2-3$ weeks ${ }^{1,3,5}$. Clinicians are challenged with providing evidence-based and effective symptom control therapies as the viral syndromes progress.

Reducing overprescribing of antibiotics for respiratory infections has been emphasized in China in the past decade to tackle antibiotic resistance ${ }^{9-12}$. Previous study showed that $93.5 \%$ of acute bronchitis visits in Chinese primary healthcare settings involved an antibiotic prescription, among which more than $50 \%$ were inappropriate ${ }^{9}$. However, studies are limited on the rates of antibiotic use and antibiotic selection for acute bronchitis in primary healthcare settings in China, as well as the detailed symptom treatment strategies for acute bronchitis. Therefore, we designed this study to evaluate outpatient antibiotic utilization, predictors of antibiotic use, and overall prescribing patterns for acute bronchitis at primary healthcare in China.

\section{RESULTS}

\section{Description of demographic characteristics}

A total of 10,678 prescriptions for acute bronchitis from 214 Chinese primary healthcare settings in 37 cities in eight provinces were eligible for inclusion in our study (Table 1). After weight adjustment, $57.3 \%$ of the prescriptions were from urban community healthcare centers and $42.5 \%$ were from rural township hospitals; $24.9 \%, 22.8 \%, 22.9 \%$, and $29.4 \%$ of the prescriptions were from the cities that in lowest to highest levels of economic status, respectively. Most prescriptions were from the eastern region $(57.4 \%)$, followed by central $(25.4 \%)$ and western region $(17.2 \%) ; 52.7 \%$ of the patients were females and $30.7 \%$ were aged 18-64 years.

\section{Antibiotic prescribing and predictors}

After weighting to better reflect the national income and urban/ rural distribution, the antibiotic prescription rate (APR) for acute bronchitis was $44.5 \%$ (unadjusted for other variables). After further adjustment (Table 1), clinicians in community centers prescribed antibiotics for acute bronchitis less frequently than those in township hospitals $(42.3 \%$ vs. $53.6 \%$, coefficients $=1.112$, $p=0.039$ ). The facilities in cities of the highest economic status had lower adjusted APR (41.1\%) than those in the lowest economic status (50.3\%) although the difference was not statistically significant (coefficients $=0.908, p=0.216$ ). Facilities located in eastern region (41.3\%) had significantly lower adjusted APR than those in central region $(56.0 \%$, coefficients $=1.148$, $p=0.019)$ and non-significantly lower APR than western region (53.4\%, coefficients $=1.122, p=0.218$ ). Economic status, patient gender, and patient age were not the significant predictors of antibiotic prescribing.

\footnotetext{
${ }^{1}$ Department of Pharmacy Administration and Clinical Pharmacy, School of Pharmaceutical Sciences, Peking University, Beijing, China. ${ }^{2}$ Department of Population Medicine, Harvard Medical School and Harvard Pilgrim Health Care Institute, Boston, MA, USA. International Research Center for Medicinal Administration, Peking University, Beijing, China. ${ }^{4}$ Clinical Epidemiology Research Center, Peking University Third Hospital, Beijing, China. ${ }^{5}$ These authors contributed equally: Xiaodong Guan, Luwen Shi.

凶email: guanxiaodong@pku.edu.cn; shiluwen211@163.com
} 
Table 1. Characteristics and antibiotic use for acute bronchitis at primary healthcare facilities in China.

\begin{tabular}{|c|c|c|c|c|c|c|c|c|}
\hline \multicolumn{9}{|l|}{ Area } \\
\hline Urban & 5369 & 50.3 & 6134.0 & 57.5 & Reference & & & 42.3 \\
\hline Rural & 5309 & 49.7 & 4542.3 & 42.5 & 1.112 & $(1.005-1.219)$ & 0.039 & 53.6 \\
\hline \multicolumn{9}{|c|}{ Economic status } \\
\hline Middle low & 4414 & 41.3 & 2435.4 & 22.8 & 0.986 & $(0.859-1.114)$ & 0.835 & 49.0 \\
\hline Middle High & 762 & 7.1 & 2447.8 & 22.9 & 0.990 & $(0.852-1.128)$ & 0.890 & 49.4 \\
\hline High & 3929 & 36.8 & 3133.6 & 29.4 & 0.908 & $(0.763-1.054)$ & 0.216 & 41.1 \\
\hline \multicolumn{9}{|l|}{ Region } \\
\hline Eastern & 6111 & 57.2 & 6132.3 & 57.4 & Reference & & & 41.3 \\
\hline Male & 5081 & 47.6 & 5045.9 & 47.3 & 0.990 & $(0.953-1.027)$ & 0.601 & 46.6 \\
\hline \multicolumn{9}{|l|}{ Patient age } \\
\hline $0-5$ & 810 & 7.6 & 746.6 & 7.0 & Reference & & & 44.3 \\
\hline $6-17$ & 487 & 4.6 & 464.2 & 4.3 & 1.058 & $(0.981-1.135)$ & 0.139 & 50.1 \\
\hline $18-64$ & 3211 & 30.1 & 3274.0 & 30.7 & 1.046 & $(0.973-1.119)$ & 0.216 & 48.9 \\
\hline $65-79$ & 1467 & 13.7 & 1447.7 & 13.6 & 1.006 & $(0.920-1.093)$ & 0.890 & 44.9 \\
\hline $80+$ & 562 & 5.3 & 532.4 & 5.0 & 0.951 & $(0.876-1.027)$ & 0.205 & 39.4 \\
\hline Unknown & 4141 & 38.8 & 4211.4 & 39.4 & 1.034 & $(0.918-1.150)$ & 0.564 & 47.7 \\
\hline Total & 10,678 & 100.0 & $10,676.3$ & 100.0 & - & - & - & - \\
\hline
\end{tabular}

\section{Distribution of prescribed antibiotic by drug classes}

Of all the antibiotic prescriptions for acute bronchitis, $12.0 \%$ contained more than one antibiotic (Table 2). Township hospitals had significantly higher multi-APR than community centers $(15.7 \%$ vs. $7.7 \%, p=0.000)$. Among all single-antibiotic prescriptions, $91.5 \%$ were broad-spectrum. Township hospitals had significantly higher rate of broad-spectrum antibiotics than community centers $(96.8 \%$ vs. $86.5 \%, p=0.000)$. The most commonly prescribed antibiotic classes in urban areas were second-generation cephalosporins (31.8\%), macrolides (19.8\%), and third-generation cephalosporins (19.6\%). In contrast, thirdgeneration cephalosporins $(23.1 \%)$ were identified as the most commonly used antibiotic in rural areas, followed by macrolides (19.4\%) and broad-spectrum penicillins (16.9\%). In both urban and rural areas, the most commonly used second-generation cephalosporins were cefuroxime $(66.2 \%$ vs. $46.1 \%$, respectively) and cefaclor ( $24.8 \%$ vs. $43.0 \%$, respectively). The most commonly used third-generation cephalosporins were cefixime, cefdinir, and ceftazidime in urban areas (31.7\%, 24.7\%, 23.3\%), and ceftazidime, cefotaxime, and ceftriaxone in rural areas $(46.9 \%, 22.8 \%, 15.7 \%)$.

\section{Antibiotic prescribing patterns and symptom management medication for adults}

Of 5240 prescriptions for acute bronchitis patients aged 18 and above, $46.4 \%$ included antibiotics and $65.2 \%$ included symptom management drugs (Table 3). Traditional Chinese Medicines for respiratory system were identified as the most commonly prescribed symptom management drug categories (35.2\%), followed by expectorants (26.8\%) and antihistamines (8.9\%). Routine treatment of uncomplicated acute bronchitis with antibiotics was not recommended in China, US, and UK. Penicillins, macrolides, cephalosporins, and fluoroquinolones were recommended as treatment options for certain patients by the Chinese national guideline. Amoxicillin, clarithromycin, and erythromycin were recommended by the UK for certain patients. Cough suppressants were recommended for cough symptom treatment in China, US, and UK, while inhaled or systemic corticosteroids for cough management were not recommended in all the countries (Supplementary Table 2).

Overall, $17.3 \%$ of the adult patients in our sample were prescribed only antibiotics for acute bronchitis, while $36.1 \%$ were prescribed only symptom management drugs, $29.1 \%$ were prescribed both antibiotics and symptom management drugs, and $17.5 \%$ were not prescribed with drugs for acute bronchitis (Table 4). The overall rate of compliance with the Chinese national clinical guideline for acute bronchitis management was $31.0 \%$. Community centers showed better compliance than the township hospitals (33.2\% vs. $28.0 \%, p=0.000$ ). The overall compliance rates of Chinese primary healthcare prescribers following clinical guidelines of the US and the UK were $18.5 \%$ and $27.4 \%$, respectively.

\section{DISCUSSION}

In light of the paucity of studies investigating detailed outpatient treatment patterns for acute bronchitis in primary healthcare settings in China, we evaluated prescribing patterns of antibiotics and symptom management medications for patients with acute bronchitis treated in 214 primary healthcare settings across China.

Our results indicated that, in 2017, Chinese primary healthcare clinicians prescribed antibiotics for nearly half of 
Table 2. Antibiotics prescribed for acute bronchitis at primary healthcare facilities in China, by urban-rural location and antibiotic type.

\begin{tabular}{|c|c|c|c|c|}
\hline \multirow[t]{2}{*}{ Antibiotic subgroup/substance } & \multicolumn{4}{|c|}{$\begin{array}{l}\text { Prescription rate } \\
\text { (weighted, \%) }^{\mathrm{a}}\end{array}$} \\
\hline & Total & Urban & Rural & $p$ \\
\hline Single-antibiotic prescriptions & 88.0 & 92.3 & 84.3 & $* * *$ \\
\hline Narrow spectrum & 8.5 & 3.2 & 13.5 & $* * *$ \\
\hline First-generation cephalosporins & 4.9 & 1.1 & 8.7 & $* * * *$ \\
\hline Narrow-spectrum penicillins & 3.1 & 2.0 & 4.1 & $* * *$ \\
\hline Nitroimidazoles & 0.4 & 0.2 & 0.7 & $* * *$ \\
\hline Broad-spectrum & 91.5 & 96.8 & 86.5 & $* * *$ \\
\hline Second-generation cephalosporins & 22.4 & 31.8 & 13.3 & $*$ \\
\hline Third-generation cephalosporins & 21.4 & 19.6 & 23.1 & $* * *$ \\
\hline Macrolides & 19.6 & 19.8 & 19.4 & $* * *$ \\
\hline Broad-spectrum penicillins & 14.7 & 12.4 & 16.9 & $* * *$ \\
\hline Quinolones & 7.2 & 7.5 & 7.0 & $* * *$ \\
\hline Lincosamides & 5.0 & 5.3 & 4.6 & $* * *$ \\
\hline Aminoglycosides & 0.7 & 0.0 & 1.4 & $* * *$ \\
\hline Other & 0.6 & 0.4 & 0.8 & $* * *$ \\
\hline Multi-antibiotic prescriptions & 12.0 & 7.7 & 15.7 & $* * *$ \\
\hline $\begin{array}{l}\text { Third-generation cephalosporins } \\
\text { +quinolones }\end{array}$ & 20.0 & 10.7 & 24.0 & $* * *$ \\
\hline $\begin{array}{l}\text { Second-generation cephalosporins } \\
\text { +quinolones }\end{array}$ & 11.5 & 18.2 & 8.5 & $* * *$ \\
\hline Broad-spectrum penicillins+quinolones & 8.5 & 3.5 & 10.7 & $* *$ \\
\hline Macrolides+quinolones & 7.2 & 18.9 & 2.1 & $* * *$ \\
\hline Macrolides+lincosamides & 6.2 & 18.2 & 0.9 & $* * *$ \\
\hline $\begin{array}{l}\text { Third-generation cephalosporins + broad- } \\
\text { spectrum penicillins }\end{array}$ & 4.6 & 0.0 & 6.7 & $* * *$ \\
\hline $\begin{array}{l}\text { Second-generation cephalosporins } \\
\text { duplications }\end{array}$ & 4.4 & 6.2 & 3.6 & \\
\hline $\begin{array}{l}\text { Second-generation+third-generation } \\
\text { cephalosporins }\end{array}$ & 4.3 & 0.0 & 6.2 & $* * *$ \\
\hline Other & 33.3 & 24.2 & 37.3 & $* *$ \\
\hline Total & 100.0 & 100.0 & 100.0 & \\
\hline
\end{tabular}

${ }^{*} p<0.05 ;{ }^{* *} p<0.01 ;{ }^{* * *} p<0.001$.

${ }^{\mathrm{a}}$ For single-antibiotic prescriptions or multi-antibiotic prescriptions, the numerators of prescription rates were the weighted number of prescriptions that containing one or multiple antibiotics, and the denominator was total weighted number of antibiotic prescriptions. For narrow spectrum and broad-spectrum antibiotics, the numerator was the weighted number of prescriptions that contained each specific antibiotic and the denominator was the weighted number of prescriptions that contained only one antibiotic. For multi-antibiotic prescriptions, the numerator was the weighted number of prescriptions that contained each specific antibiotic combination and the denominator was the weighted number of prescriptions that contained multiple antibiotics.

outpatients with acute bronchitis, which was a lower rate than those in the studies conducted in Italy $(73.5 \%)^{13}$, US $(74 \%)^{14}$, Belgium $(69.0 \%)^{15}$, Spain $(58.6 \%)^{16}$, Japan $(52.8 \%)^{17}$, and Netherlands $(51.0 \%)^{18}$, and was also lower than previous studies in China (77.5-95.2\%) ${ }^{9,11}$. However, our rate was slightly higher than the rate showed in the study conducted in Swiss $(41.5 \%)^{19}$. One study conducted in England found that $82 \%$ of the consultations for acute bronchitis resulted in an antibiotic prescription on the same day, among which only $13 \%$ of the patients was necessary for antibiotic treatment according to guidelines and expert elicitations ${ }^{20}$. Another study showed that
$85.6 \%$ of the antibiotic treatment duration exceeded guideline recommendations in primary care in England ${ }^{7}$. The relatively lower prevalence in our study could be due to the changing patterns of antibiotic use in China ${ }^{21}$, or to our limiting inclusion of acute bronchitis cases without any other infectious diagnosis. The higher rates of antibiotic prescribing in previous studies may be driven by misconceptions of clinicians and patients ${ }^{22,23}$. A Cochrane review suggested that antibiotics were minimally effective for acute bronchitis, with a half-day reduction in cough and no reduction in functional impairment compared to placebo, as well as increasing rates of adverse events ${ }^{3}$. Public perception/misconception of antibiotic efficacy drove inappropriate demand for utilizing antibiotics in the primary outpatient setting in China ${ }^{24}$. Surveys showed that $60 \%$ of the population believed that antibiotics were effective for the treatment of acute bronchitis ${ }^{25}$, a belief that varied by educational level $^{26}$. This may partially explain the higher antibiotic prescribing rate in rural and the less developed western region in our study.

Among all single-antibiotic prescriptions, 91.5\% were broadspectrum formulations, and second- and third-generation cephalosporins were the most prescribed categories. This was consistent with previous findings in Chinese hospitals for treating respiratory tract infections ${ }^{9,10}$. This preference for broad-spectrum cephalosporins was also found in the US and European countries ${ }^{27}$. As noted in previous studies, cephalosporins should be avoided when a narrow-spectrum antibiotic would be effective ${ }^{28}$. Inappropriate antibiotic use accelerated the development of antibiotic resistance such as methicillinresistant staphylococci ${ }^{29}$. Cephalosporin overuse in Chinese primary healthcare outpatient settings may be largely due to the requirement of mandatory skin testing before prescribing either oral or injectable penicillins for all patients ${ }^{30}$. Ideally, skin testing can increase the number of incidents in which penicillin can be safely administered rather than alternative broadspectrum antibiotics ${ }^{31}$. However, in clinical practice, overestimation of penicillin allergy rates $^{32,33}$, along with the inconvenience and unreliable results of penicillin skin testing, may actually impel some primary healthcare clinicians to replace penicillins with cephalosporins or other broadspectrum antibiotics ${ }^{31,33}$.

Supportive care and symptom management were the mainstay of treatment for adult patients with acute bronchi$\mathrm{tis}^{34}$. Our study provided a comprehensive overview of prescribing patterns of symptom management drugs for acute bronchitis patients in Chinese primary healthcare settings; however, evidence supporting these symptomatic therapies was limited according to national clinical guidelines ${ }^{5,34,35}$. For example, inhaled or systemic corticosteroids for cough management were not recommended as there were no data to support their therapeutic effect for acute cough caused by acute bronchitis ${ }^{34-36}$. Insufficient quality data were support to recommend the routine use of Chinese herbs for acute bronchitis and other respiratory conditions according to a Cochrane review ${ }^{37}$.

Barriers to evidence-based treatment of acute bronchitis in Chinese primary healthcare settings include three main issues. First, most primary healthcare clinicians in China lacked of training for evidence-based thinking, and they mainly practiced based on their experience without paying attention to the latest evidence ${ }^{38}$. Second, even if familiar with evidence-based practice, primary healthcare clinicians may have difficulties getting access to updated clinical guidelines, as most of the guidelines were published in academic journals and cannot be obtained for free. Third, Chinese guidelines were sometimes vaguer in expression and more liberal in medication selection than those in other countries (Supplementary Table 2), which made it harder for primary clinicians to understand and to follow the guidelines during daily practice. Furthermore, systems for 
Table 3. Prescribing of antibiotic and symptom management medications for adult acute bronchitis at primary healthcare facilities in China, by urban-rural location and whether medications were included in clinical guidelines.

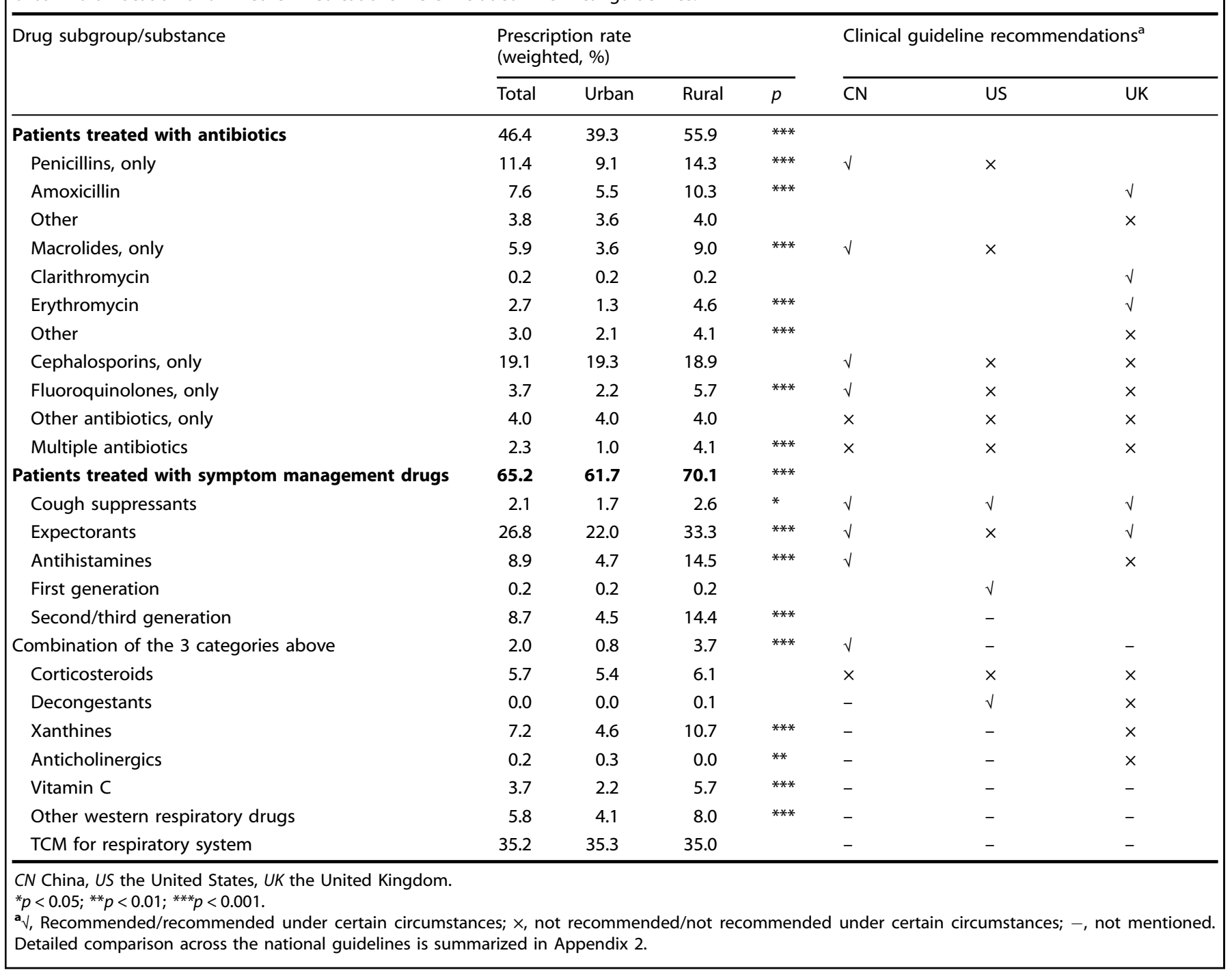

management and supervision of antibiotic utilization in Chinese primary healthcare settings were generally not in place. Routine national antimicrobial utilization and antimicrobial resistance surveillance networks for large hospitals in China operated for years ${ }^{39,40}$, but similar systems was not established in primary healthcare settings in China ${ }^{7}$.

Our study found that prescribing of antibiotics, especially broad-spectrum ones like cephalosporins, for acute bronchitis remained common in Chinese primary healthcare settings. Antibiotic stewardship and interventions to improve the recognition of inappropriate antibiotic prescribing were urgently needed. Efficient electronic practice advisory tools in prescribing systems ${ }^{4}$, prescriber education ${ }^{12}$, public reporting ${ }^{11}$, shared decision making ${ }^{41}$, delayed antibiotic prescriptions ${ }^{42}$, and involvement of multidisciplinary teams for audit and feedback $^{12}$ were effective measures to improve antibiotic prescribing in previous studies. Primary healthcare clinicians in China may have difficulties in accessing, understanding, and following clinical guidelines in practice. Therefore, tailored training and continuous education for primary healthcare clinicians that targeted at evidence-based treatment based on clinical guidelines could contribute to promoting prescribing quality. In addition, routine national antibiotic stewardship systems based on real-time utilization data would be beneficial in improving antibiotic use in Chinese primary healthcare settings.

Several limitations to this study should be noted. First, we selected acute bronchitis prescriptions from a national survey sample, which was designed to cover the full range of primary care diagnoses. The study sample was not designed to be fully representative of all acute bronchitis patients at primary healthcare settings in China. Second, we adopted overall population counts by using levels of economic status and urban-rural areas as weights to adjust the analyses to better reflect national data. However, the prevalence of acute bronchitis was not equally distributed in all regions of China, and we weighted our estimates based on population. This may lead to over- or under-weighting of the sample estimates. However, given that prescriptions were sampled consistently in all facilities, the prevalence of acute bronchitis cases in the sample should be reasonably representative of their overall prevalence at the time of the study. Third, due to the limitations of the data access, many factors that may affect the quality of prescribing, such as characteristics and knowledge of clinicians, laboratory tests, 


\begin{tabular}{|c|c|c|c|c|c|c|c|c|c|c|c|c|c|c|c|c|}
\hline Antibiotics, only & 17.3 & 17.2 & 17.4 & & 0.8 & 1.5 & 0.0 & * & 0.0 & 0.0 & 0.0 & - & 0.0 & 0.0 & 0.0 & - \\
\hline Symptom management drugs, only & 36.1 & 39.5 & 31.5 & $* * *$ & 37.0 & 29.9 & 49.1 & $* * *$ & 2.7 & 3.0 & 2.3 & & 27.5 & 24.1 & 33.3 & $* * *$ \\
\hline Total & 100.0 & 100.0 & 100.0 & - & 31.0 & 33.2 & 28.0 & $* * *$ & 18.5 & 22.3 & 13.3 & $* * *$ & 27.4 & 30.7 & 23.1 & $* * *$ \\
\hline
\end{tabular}

patient socio-economic characteristics, medical history, drug affordability, cigarette smoking, and environmental pollution were not measured. Fourth, although we excluded the patients who also had a diagnosis of other infections together with acute bronchitis, we cannot assure that the drugs were prescribed only for acute bronchitis.

In conclusion, clinicians prescribed antibiotics for nearly half of outpatients with acute bronchitis in Chinese primary healthcare settings, a level far higher than indicated by guidelines, with particular overuse of broad-spectrum antibiotics. Medications for symptom management were also commonly used in practice. In all, more than two-thirds of the acute bronchitis treatments for adults were not consistent with guideline recommendations. Targeted interventions to improve primary healthcare practice and systems to increase detection of inappropriate antibiotic prescribing were urgently needed for Chinese primary clinicians, especially in western rural areas.

\section{METHODS}

\section{Study design}

We conducted a nationwide cross-sectional survey of primary healthcare settings in China to collect outpatient prescriptions from January 2017 to December 2017. Ethics approval was obtained from Peking University Institution Review Board.

\section{Sampling and data collection}

We systematically selected community healthcare centers in urban areas and township hospitals in rural areas across China. First, we classified all 408 cities in China into four levels of economic status according to GDP per capita in 2016. We then selected nine provinces (East region: Beijing, Shandong, Guangdong; Central region: Jilin, Anhui, Jiangxi; West region: Qinghai, Sichuan, Yunnan) out of 31 provinces in mainland China to conduct the survey. Among these provinces, 42 cities were randomly selected according to the four economic strata. Finally, in each selected city, two community healthcare centers and nine township hospitals were selected randomly based on the ratio of these two facility types nationally in 2017 (ref. ${ }^{43}$ ).

In each sample facility, 50 outpatient prescriptions were randomly selected from patient encounters that took place on the second Tuesday of each month. Prescription data including visit date, patient demographic characteristics, diagnoses, and medications were digitally extracted from outpatient records and verified by two investigators.

\section{Inclusion criteria}

Patients diagnosed with acute bronchitis were eligible for this study. Patients who also diagnosed with other infections were excluded (Supplementary Table 1).

\section{Measurements}

We classified antibiotics according to Anatomical Therapeutic and Chemical classification $\mathrm{J} 01$ (ref. ${ }^{44}$ ). Narrow-spectrum antibiotics consisted of narrow-spectrum penicillins, first-generation cephalosporins, nitroimidazoles, tetracyclines, and sulfonamides. Broad-spectrum antibiotics included broad-spectrum penicillins, advanced-generation cephalosporins, macrolides, quinolones, lincosamides, aminoglycosides (except for streptomycin), and other antibiotics (e.g., fosfomycin) ${ }^{45}$.

The primary outcome was APR. The numerator was the weighted number of acute bronchitis prescriptions that contained at least one antibiotic, and the denominator was the total weighted number of acute bronchitis prescriptions. Secondary outcomes included the prescription rate of different antibiotic classes, the proportions of different treatments for acute bronchitis, and the overall guideline compliance rate. We presented the detailed comparisons of national guidelines for treatment strategies of adult acute bronchitis in China, US, and UK in Supplementary Table 2, and the definition of our measure of overall guideline compliance in Supplementary Table 3. Independent variables in our analysis included economic status and region of the city, urban/rural location of the clinic, and patient gender and age.

\section{Statistical analysis}

STATA 14.0 was used for all statistical analysis. We used generalized estimating equations to generate population-weighted comparisons, standardized margins to calculate mean adjusted proportions, and chi square test to compare the urban-rural differences. We weighted sample estimates to reflect the distribution of economic level and urban-rural status in the national population. The level of statistical significance was defined as $p<0.05$ (two sided).

\section{Reporting summary}

Further information on research design is available in the Nature Research Reporting Summary linked to this article.

\section{DATA AVAILABILITY}

Data of this study are available upon reasonable request from corresponding author. The data are not publicly available due to restrictions, e.g., their containing information that could compromise the privacy of research participants.

\section{CODE AVAILABILITY}

Code of this study are available upon reasonable request from corresponding author.

Received: 9 September 2020; Accepted: 29 March 2021; Published online: 10 May 2021 


\section{REFERENCES}

1. Albert, R. H. Diagnosis and treatment of acute bronchitis. Am. Fam. Phys. 82, 1345-1350 (2010).

2. Singh, A., Avula, A. \& Zahn, E. In StatPearls (StatPearls Publishing, 2020).

3. Smith, S. M., Fahey, T., Smucny, J. \& Becker, L. A. Antibiotics for acute bronchitis. Cochrane Database Syst. Rev. 6, CD000245 (2017).

4. Pagels, C. M., Dilworth, T. J., Fehrenbacher, L., Singh, M. \& Brummitt, C. F. Impact of an electronic best-practice advisory in combination with prescriber education on antibiotic prescribing for ambulatory adults with acute, uncomplicated bronchitis within a large integrated health system. Infect. Control Hosp. Epidemiol. 40, 1348-1355 (2019).

5. Harris, A. M., Hicks, L. A. \& Qaseem, A. \& High Value Care Task Force of the American College of Physicians and for the Centers for Disease Control and Prevention. Appropriate antibiotic use for acute respiratory tract infection in adults: advice for high-value care from the American College of Physicians and the Centers for Disease Control and Prevention. Ann. Intern. Med. 164, 425-434 (2016).

6. Smith, M. P. et al. Acute cough due to acute bronchitis in immunocompetent adult outpatients: CHEST Expert Panel Report. Chest 157, 1256-1265 (2020).

7. Pouwels, K. B. et al. Duration of antibiotic treatment for common infections in English primary care: cross sectional analysis and comparison with guidelines. BMJ 364, 1440 (2019).

8. Centers for Disease Control and Prevention. Antibiotic Resistance Report. Vol. 2019. https://www.cdc.gov/drugresistance/pdf/threats-report/2019-ar-threats-report508.pdf (CDC, 2019).

9. Wang, J., Wang, P., Wang, X., Zheng, Y. \& Xiao, Y. Use and prescription of antibiotics in primary health care settings in China. JAMA Intern. Med. 174, 1914-1920 (2014).

10. $\mathrm{Wu}, \mathrm{Y}$. et al. Prescription of antibacterial agents for acute upper respiratory tract infections in Beijing, 2010-2012. Eur. J. Clin. Pharmacol. 72, 359-364 (2016).

11. Tang, Y., Liu, C. \& Zhang, X. Public reporting as a prescriptions quality improvement measure in primary care settings in China: variations in effects associated with diagnoses. Sci. Rep. 6, 39361 (2016).

12. Wei, X. et al. Effect of a training and educational intervention for physicians and caregivers on antibiotic prescribing for upper respiratory tract infections in children at primary care facilities in rural China: a cluster-randomised controlled trial. The Lancet. Lancet Glob. Health 5, e1258-e1267 (2017).

13. Bianco, A., Papadopoli, R., Mascaro, V., Pileggi, C. \& Pavia, M. Antibiotic prescriptions to adults with acute respiratory tract infections by Italian general practitioners. Infect. Drug Resist. 11, 2199-2205 (2018).

14. Barnett, M. L. \& Linder, J. A. Antibiotic prescribing for adults with acute bronchitis in the United States, 1996-2010. JAMA 311, 2020-2022 (2014).

15. Colliers, A. et al. Antibiotic prescribing quality in out-of-hours primary care and critical appraisal of disease-specific quality indicators. Antibiotics 8, 79 (2019).

16. March-López, P. et al. Impact of a multifaceted antimicrobial stewardship intervention in a primary health care area: a quasi-experimental study. Front. Pharmacol. 11, 398 (2020).

17. Hashimoto, $\mathrm{H}$. et al. Antibiotic prescription among outpatients in a prefecture of Japan, 2012-2013: a retrospective claims database study. BMJ Open 9, e026251 (2019).

18. Hek, K., van Esch, T., Lambooij, A., Weesie, Y. M. \& van Dijk, L. Guideline adherence in antibiotic prescribing to patients with respiratory diseases in primary care: prevalence and practice variation. Antibiotics 9, 571 (2020).

19. Glinz, D. et al. Quality of antibiotic prescribing of Swiss primary care physicians with high prescription rates: a nationwide survey. J. Antimicrob. Chemother. 72, 3205-3212 (2017).

20. Pouwels, K. B., Dolk, F., Smith, D., Robotham, J. V. \& Smieszek, T. Actual versus 'ideal' antibiotic prescribing for common conditions in English primary care. $J$. Antimicrob. Chemother. 73, 19-26 (2018).

21. Wirtz, V. J. et al. Essential medicines for universal health coverage. Lancet 389, 403-476 (2017).

22. McKay, R., Mah, A., Law, M. R., McGrail, K. \& Patrick, D. M. Systematic review of factors associated with antibiotic prescribing for respiratory tract infections. Antimicrob. Agents Chemother. 60, 4106-4118 (2016).

23. Dempsey, P. P., Businger, A. C., Whaley, L. E., Gagne, J. J. \& Linder, J. A. Primary care clinicians' perceptions about antibiotic prescribing for acute bronchitis: a qualitative study. BMC Fam. Pract. 15, 194 (2014).

24. Lin, L., Sun, R., Yao, T., Zhou, X. \& Harbarth, S. Factors influencing inappropriate use of antibiotics in outpatient and community settings in China: a mixedmethods systematic review. BMJ Glob. Health 5, e003599 (2020).

25. Cals, J. W. et al. Public beliefs on antibiotics and respiratory tract infections: an internet-based questionnaire study. Br. J. Gen. Pract. 57, 942-947 (2007).

26. Rousounidis, A. et al. Descriptive study on parents' knowledge, attitudes and practices on antibiotic use and misuse in children with upper respiratory tract infections in Cyprus. Int. J. Environ. Res. Public Health 8, 3246-3262 (2011).
27. Shapiro, D. J., Hicks, L. A., Pavia, A. T. \& Hersh, A. L. Antibiotic prescribing for adults in ambulatory care in the USA, 2007-09. J. Antimicrob. Chemother. 69, 234-240 (2014).

28. Appropriate use of cephalosporins. BPJ Issue 41, Vol. 2020. https://bpac.org.nz/ BPJ/2011/december/cephalosporins.aspx (2011).

29. Su, T., Broekhuizen, B., Verheij, T. \& Rockmann, H. The impact of penicillin allergy labels on antibiotic and health care use in primary care: a retrospective cohort study. Clin. Transl. Allergy 7, 18 (2017).

30. Wang, C. N. et al. Pediatric antibiotic prescribing in china according to the 2019 World Health Organization Access, Watch, and Reserve (AWaRe) antibiotic categories. J. Pediatr. 220, 125-131.e5 (2020).

31. Bhattacharya, S. The facts about penicillin allergy: a review. J. Adv. Pharm. Technol. Res. 1, 11-17 (2010).

32. Joint Task Force on Practice Parameters. et al. Drug allergy: an updated practice parameter. Ann. Allergy, Asthma Immunol. 105, 259-273 (2010).

33. Meng, J. \& Wang, L. Penicillin allergy and diagnostic methods. Chin. J. Allergy Clin. Immunol. 10, 412-420 (2016).

34. Kinkade, S. \& Long, N. A. Acute bronchitis. Am. Fam. physician 94, 560-565 (2016).

35. National Institute for Health and Care Excellence. Cough (Acute): Antimicrobial Prescribing. Vol. 2019. www.nice.org.uk/guidance/ng120 (2019).

36. Chinese Medical Association. et al. Guideline for primary care of acute tracheobronchitis (2018). Chin. J. Gen. Practitioners 18, 314-317 (2019).

37. Jiang, L., Li, K. \& Wu, T. Chinese medicinal herbs for acute bronchitis. Cochrane Database Syst. Rev. 2012, CD004560 (2012).

38. Wushouer, H. et al. The impact of physicians' knowledge on outpatient antibiotic use: evidence from China's county hospitals. Medicine 99, e18852 (2020).

39. Center for Antibacterial Surveillance. Vol. 2020. http://y.chinadtc.org.cn/login (2020).

40. China Antimicrobial Resistance Surveillance System. Vol. 2020. http://www.carss.cn/ (2020).

41. Spurling, G. K., Del Mar, C. B., Dooley, L., Foxlee, R. \& Farley, R. Delayed antibiotic prescriptions for respiratory infections. Cochrane database Syst. Rev. 9, CD004417 (2017).

42. Ivers, N. et al. Audit and feedback: effects on professional practice and healthcare outcomes. Cochrane Database Syst. Rev. CD000259 (2012).

43. National Health Commission. 2017 China Health and Family Planning Statistical Yearbook. 1st edn (Peking Union Medical College Press, 2017) (in Chinese).

44. World Health Organization. Guidelines for ATC Classification and DDD Assignment Vol. 2020. https://www.whocc.no/filearchive/publications/2020_guidelines_web. pdf (WHO, 2018).

45. Havers, F. P. et al. Outpatient antibiotic prescribing for acute respiratory infections during influenza seasons. JAMA Netw. Open 1, e180243 (2018).

\section{ACKNOWLEDGEMENTS}

We thank Dr. Fang Zhang, Assistant Professor, Department of Population Medicine, Harvard Medical School and Harvard Pilgrim Health Care Institute, for providing technical support for the statistical analysis. Our sincere appreciation to National Heath Commission of China, Department of Drug Policy and Essential Medicine for providing great support for data collection of this study. This work was supported by China Medical Board [grant number: 89200672BC1], National Natural Science Foundation of China [grant number: 71774005] and China Scholarship Council [grant number: 201906010364] to M.F.

\section{AUTHOR CONTRIBUTIONS}

Study concept and design: M.F., X.G., D.R.-D., L.S., H.W. Acquisition, analysis, or interpretation of data: M.F., X.G. and L.S. Drafting of the manuscript: M.F., X.G., D.R.-D., and H.W. L.H., Critical revision of the manuscript for important intellectual content: X.G., D.R.-D. Statistical analysis: M.F. Administrative, technical, or material support: N.L., D.R.-D., L.H. Supervision: L.S.

\section{COMPETING INTERESTS}

The authors declare no competing interests.

\section{ADDITIONAL INFORMATION}

Supplementary information The online version contains supplementary material available at https://doi.org/10.1038/s41533-021-00234-y.

Correspondence and requests for materials should be addressed to X.G. or L.S. 
Reprints and permission information is available at http://www.nature.com/ reprints

Publisher's note Springer Nature remains neutral with regard to jurisdictional claims in published maps and institutional affiliations.

(i) Open Access This article is licensed under a Creative Commons Attribution 4.0 International License, which permits use, sharing, adaptation, distribution and reproduction in any medium or format, as long as you give appropriate credit to the original author(s) and the source, provide a link to the Creative Commons license, and indicate if changes were made. The images or other third party material in this article are included in the article's Creative Commons license, unless indicated otherwise in a credit line to the material. If material is not included in the article's Creative Commons license and your intended use is not permitted by statutory regulation or exceeds the permitted use, you will need to obtain permission directly from the copyright holder. To view a copy of this license, visit http://creativecommons. org/licenses/by/4.0/.

(c) The Author(s) 2021 